\title{
Ultrafast carrier's dynamics with scattering rate saturation in Ge thinfilms
}

This paper was downloaded from TechRxiv (https://www.techrxiv.org).

\section{LICENSE}

CC BY 4.0

SUBMISSION DATE / POSTED DATE

$09-11-2021 / 10-11-2021$

\section{CITATION}

Saxena, Praveen; Gupta, Fanish Kumar; Srivastava, Anshika; Srivastava, Pankaj; Saxena, Anshu (2021): Ultrafast carrier's dynamics with scattering rate saturation in Ge thinfilms. TechRxiv. Preprint. https://doi.org/10.36227/techrxiv.16960813.v1

$\mathrm{DOI}$

10.36227/techrxiv.16960813.v1 


\title{
Ultrafast carrier's dynamics with scattering rate saturation in Ge thinfilms
}

\author{
P. K. Saxena ${ }^{1}$, F. K. Gupta ${ }^{1}$, A. Srivastava ${ }^{1}$, P. Srivastava ${ }^{1}$ and Anshu Saxena ${ }^{1}$ \\ ${ }^{1}$ Tech Next Lab Pvt Ltd, \\ Lucknow, India, \\ email: a.saxena@technextlab.com
}

\begin{abstract}
An innovative theoretical approach for deeper understanding of the ultrafast spectroscopy experiments through solution of the Boltzmann transport equation coupled with various nonlinear scattering mechanisms and overcoming the limitations poses by DFT, RT-TDDFT and molecular based methods, is reported. A clear advantage of the real-time approach is that it does not make a priori assumptions about specific scatterings, relaxation mechanisms and has capabilities to capture the full real-time carrier's dynamics, including the superposition of all electron-electron, electron-lattice and electron-phonon scatterings etc. No such method with advances in theoretical treatments to explain ultrafast spectroscopy has been reported previously as per the author's knowledge.
\end{abstract}

\section{Introduction}

Terahertz (THz) spectroscopy is a key technology for contact-less conductivity measurements of metals, semiconductors, 2D materials, and superconductors. The interaction of $\mathrm{THz}$ pulses with charge carriers in semiconductor materials are responsible for inter- and intravalley carrier transitions and provide valuable information about the carrier and quasi-particle dynamics in semiconductors [1] - [6].

The typical ultrafast pump-probe experiments are carried out by generation of hot carriers with optical photon energies exceeding the band gap. The $\mathrm{THz}$ probe pulses are used to predict the hot carrier's dynamics after the excitation process. However, this method suffers with several limitations and is incapable to accurately predict the hot carrier's dynamics in bulk and nano- materials through optical excitation method [1].

Several theoretical treatments for predictions of $\mathrm{THz}$ conductivities in thin films and nano-materials have been proposed. The Drude model based on confined gas of classical non-interacting electrons requires several fitting parameters in order to calibrate the experimental results. Also, the incapability of Drude model to explain the lowfrequency conductivities suppression also poses significant challenges, especially for nano-systems where physical boundaries on the nano-scale provide a conceptually clear source of directionally biased carrier scattering mechanisms. [6] - [9]. On the other hand, explicit first-principles (DFT) based studies of the real-time electron thermalization dynamics are much less common and have only been reported for laser-excited nickel, chromium, and copper, using real time time-domain density functional theory (RT-TDDFT) [2], [9]. The current state-of-the-art approach is the solution of the Boltzmann transport equation (BTE) [2]. However, the BTE solution through Monte Carlo (MC) technique involves relaxation time approximation (RTA) approach in which the carrier scattering rates are chosen as fitting parameter on the arbitrary basis without giving any physics based justification [2], [6], [10]. Also, the impact of various types of scattering mechanisms responsible for conductivity degradation is nondistinguishable through RTA based approach [10].

To address above and other issues mentioned elsewhere in ref [2], authors are reporting an innovative solution with a more solid atomistic physics based foundations to study the carriers dynamics of hot electrons. The Ge thin film is chosen due to its material optical properties (high refractive indices and large third-order nonlinearities) and its applications, e.g. Ge based core optical Fibre (GCOF) exhibit more advantages compared to silica-based optical fibers [11]-[12]. The proposed $\mathrm{THz}$ spectroscopy technique is capable to do similar analysis with any group IV, III-V and II-VI semiconductors. The peak THz field strengths $150 \mathrm{kV} / \mathrm{cm}$ is used in current study which is comparable to nonlinear semiconductor devices e.g. Gunn and tunnel diodes. The impact of the $\mathrm{THz}$ field on the carriers transportation have been analyzed in details for better understanding of the hot electrons dynamics on three valleys i.e. $\Gamma, \mathrm{L}$ and $\mathrm{X}$ respectively. The Monte Carlo (MC) technique is used for the solution of coupled BTE including various types of non-linear scattering mechanisms i.e. impact ionization, acoustic, intervalley and carrier-carrier scatterings without choosing any fitting parameters [13]. As per the author's knowledge, the innovative technique with advances in theoretical treatments to explain ultrafast spectroscopy has been reported for the first time without using any fitting parameters. All results reported in current manuscript can be reproduced by users [13].

\section{Technique}

The Boltzmann transport equation (BTE) under nonequilibrium conditions is used to predict the hot carrier's dynamics on three valleys on full electronic structure. The inhouse developed algorithm based on Monte Carlo technique is used for solution of the BTE [14]. The Ge material parameters used in current analysis are tabulated in Table 1. The carrier's transportation on non-parabolic $\Gamma, \mathrm{L}$ and $\mathrm{X}$ valleys is initiating with the equal number of carriers distribution in each valleys. In absence of $\mathrm{THz}$ field, carriers 
attain equilibrium to suppress the possibility of any kind of intrinsic noise presence. Most of the high energy valley electrons transfer itself to lower energy valley. The free carriers interact strongly with $\mathrm{THz}$ field here the single-cycle $\mathrm{THz}$ pulses is generated with delay of $3 / 4 \mathrm{~T}$, where $\mathrm{T}$ is the time period of pulse. The free carrier absorption responsible for changes in carrier mobility and scattering rates is monitored for each carrier [13]-[14]. The THz pulse can be mathematically written as;

$E(t)=E_{0} \cos (2 \pi f p \mathrm{~d} t)$

Here, $E_{0}$ is the peak amplitude of the driving $\mathrm{THz}$ field, $\mathrm{f}$ is $\mathrm{THz}$ frequency, $\mathrm{p}$ is the number of total time steps and $\mathrm{dt}$ is the time step.

Under the influence of $\mathrm{THz}$ field, the electrons free flight duration is chosen with a probability distribution function which is determined by the various non-linear scattering probabilities. The various scattering rates are given in Appendix A. The change in carrier's momentum as a function of $\mathrm{THz}$ frequencies is given as;

$\mathrm{k}(\mathrm{dt})=\mathrm{k}(0)-\frac{q E(t) \mathrm{dt}}{\hbar}$

Here $\mathrm{k}(0)$ is the initial wave vector, $\mathrm{h}$ is Planck's constant, e is the electronic charge and $\mathrm{E}$ is the time dependent $\mathrm{THz}$ field [13].

The total force acting on an electron at time dt is equal to the product of time dependent $\mathrm{THz}$ field $\mathrm{E}$ and electronic charge $\mathrm{q}$ and is given as,

$F=q E(t)=\frac{d p}{d t}=\hbar \frac{d k}{d t}$

The change in carrier momentum is dependent on the scattering rate $\Gamma[\mathrm{k}(t)]$ probabilities. $\Gamma[\mathrm{k}(t)]$ is defined that a carrier has not suffered a collision after a time $t$ ' and is given as $\exp \left(-\int_{0}^{t} \Gamma\left[\mathbf{k}\left(t^{\prime}\right)\right] d t^{\prime}\right)$.

However, the scattering probability in the time duration $d t$ after a free flight of time $t$ is given by the joint probability as;

$P(t) d t=\Gamma[\mathbf{k}(t)] \exp \left[-\int_{0}^{t} \Gamma\left[\mathbf{k}\left(t^{\prime}\right)\right] d t^{\prime}\right] d t$

The $P(t)$ probability density function decides the random flight times chosen. It can be demonstrated through an example, the uniformly distributed random numbers remain in the range $[0,1]$. By using a direct method random flight times which can be sampled from $P(t)$ is

$r=\int_{0}^{t_{r}} P(t) d t$

Here $r$ is a uniformly distributed random number and $t_{\mathrm{r}}$ is the desired free flight time. After putting the value of $\mathrm{P}(\mathrm{t})$ from Eq. (4) and integrating, the relation is

$r=1-\exp \left[-\int_{0}^{t_{r}} \Gamma\left[\mathbf{k}\left(t^{\prime}\right)\right] d t^{\prime}\right]$.

The $r$ and 1-r are statistically identical therefore Eq. (6) is simplified to $-\ln r=\int_{0}^{t} \Gamma\left[\mathbf{k}\left(t^{\prime}\right)\right] d t^{\prime}$

Eq. (7) is used to generate the random free flight time for each electron after each scattering event. It results in a random walk process associated with the underlying atomistic particle distribution function. If the external driving force field is absent (e.g. in ultrafast photo-excitation experiments, with no applied bias), the value of $\mathrm{k}$ depends only between scattering events, the time dependency vanishes. To avoid this situation a fictitious scattering mechanism term called self-scattering is introduced. The self-scattering rate is kept to adjust itself in such a way that the total scattering rate remains constant in time [12].

$\Gamma=\Gamma\left[\mathbf{k}\left(t^{\prime}\right)\right]+\Gamma_{\text {self }}\left[\mathbf{k}\left(t^{\prime}\right)\right]$

where $\Gamma_{\text {self }}\left[\mathrm{k}\left(t^{\prime}\right)\right]$ is the self-scattering rate and itself is defined such that the final state before and after scattering remains identical. Hence, it has no effect on the free flight trajectory of a particle when selected as the terminating scattering mechanism. The free flight time is given by

$t_{r}=-\frac{1}{\Gamma} \ln r$.

The above algorithm successfully tracks the steady-state behavior of a single carrier with many scattering events. To implement transient behavior which require a synchronous ensemble of electrons, i.e. the above algorithms is repeated for every electron of the ensemble until the simulation completed in time step dt. Ultrafast photo-excitation theoretical experiment uses the single-cycle $\mathrm{THz}$ pulses of various pulse widths applied in the y direction, the velocity of each particle is recorded at each time step, where y-component of carrier drift velocity at the $\mathrm{p}^{\text {th }}$ time step is given by

$v_{y, p}=v_{y, p-1}+\frac{e E_{0} d t}{m^{*}} \cos (2 \pi f p d t)$

Here, $v_{y, n-1}$ is the $y$-component of the velocity at the previous time-step. The proposed model has flexibility for application of $\mathrm{THz}$ pulses coupling in any direction i.e. $\mathrm{x}, \mathrm{y}, \mathrm{z}$ of thin films. The transient carriers drift velocity is estimated by the ensemble average of the velocity component at the $\mathrm{p}^{\text {th }}$ time step and is;

$v_{d}=\frac{1}{N} \sum_{j=1}^{N} v_{y}^{j}(p d t)$

Here, $\mathrm{p}$ is the number of total time steps, $\mathrm{dt}$ is the time step, e $\mathrm{N}$ is the number of simulated particles and $\mathrm{j}$ labels the particles in the ensemble.

The time domain current density, $\mathrm{J}(\mathrm{t})$ is calculated using drift velocity at every time step and is given by [13]

$J(t)=N e v_{d}(t) \alpha_{n}(E) L_{e f f}$

Here, $\mathrm{N}$ is the total number of carriers, e is electronic charge and $\alpha_{\mathrm{n}}$ is the impact ionization coefficient expressed as a function of peak $\mathrm{THz}$ field. $\mathrm{L}_{\text {eff }}$ is the characteristic length of the high field region [15] - [17]. 
The frequency dependent complex conductivity is computed through Fourier transformation method and is given as,

$\sigma(\omega)=\frac{\sum J(t)(\cos \omega t+i \sin \omega t)}{\sum E(\cos \sin \omega t)}$

The absorption coefficient is given as,

$\alpha(\omega)=\frac{4 \pi \sigma(\omega)}{n_{r} c}$

Here, $n_{r}[12]$ is the real refractive index and $c$ is the velocity of light.

\section{Results \& Discussion}

An theoretical solution for successful demonstration of the real time $\mathrm{THz}$ spectroscopy experiments has been proposed on atomistic scale applicable for most of the semiconductors. The innovative in-house developed algorithms are capable to obtain physical parameters associated with ultrafast carrier transportation with inclusion of various nonlinear scattering mechanisms without assuming any fitting parameters. To observe the material optical properties of n-type $\left(5 \times 10^{20} \mathrm{~m}^{3}\right)$ Ge thin film is used. All the material parameters used in current study are listed in Table 1.

The peak $\mathrm{THz}$ field strengths of 1,5 and $15 \mathrm{MV} / \mathrm{m}$ are used [1]. THz pulses interact strongly with the electrons enabling them to undergo intra- and inter valley transitions. The various scattering mechanisms interrupt the carrier free flights. Acoustic [20], intervalley [20], ionized impurity [20][21], carrier-carrier [22] and impact ionization [15]-[17] scattering mechanisms are considered here and found to play a deciding role in free carrier absorption mechanism. The various scattering rates are given in Appendix A. The changes in carrier mobility and scattering rates have been monitored by the application of the single-cycle THz pulses [23].

Fig. 1 demonstrated the $\mathrm{THz}$ absorption spectrum of n-type Ge thin film at room temperature (294K). Equal numbers of electrons (20k are total number of electrons) are initialized in each $\mathrm{L}, \Gamma$ and $\mathrm{X}$ valleys. The $\mathrm{THz}$ pulses of different frequencies (Eqn. 3) have been used to study the hot carriers dynamics with different delay times $(=3 / 4 \mathrm{~T}$, with $\mathrm{T}$ time period of each pulse) to suppress the intrinsic noise. In absence of $\mathrm{THz}$ field the electrons start transferring from higher energy levels i.e. from $\Gamma$ and $X$ valleys to lower energy level $L$ valley and $X$ to $\Gamma$ valley as well. In case of Ge, $\mathrm{L}$ valley is the lowest energy electron occupation level. The single cycle $\mathrm{THz}$ pulse at $0.25 \mathrm{THz}$ frequency with peak field strength $150 \mathrm{MV} / \mathrm{m}$ ramping from minimum to maximum and maximum to minimum, the hot electrons excited from Lvalley to X-valley and $\Gamma$-valley as clearly depicted from Fig.3 where the red, blue and green curves are showing electrons transition in $\Gamma$-, L- and $\mathrm{X}$-valleys respectively. The free carriers absorb $\mathrm{THz}$ energy and reach at highest energy level. The absorption curves in Fig.1 show strong saturation over the frequency range between $1.0-2.0 \mathrm{THz}$, however in the range of $0.25-1.0 \mathrm{THz}$ at peak field intensity $\sim 1 \mathrm{MV} / \mathrm{m}(7 \%)$, the absorption curve is following the Drude type nature. At high peak $\mathrm{THz}$ field strengths i.e. 5 (33\%) and 15 (100\%)
$\mathrm{MV} / \mathrm{m}$, strong saturation of absorption curve over the entire $(0.25-2.0) \mathrm{THz}$ frequency range is observed. The absorption spectrum curve for $7 \%, 33 \%$ and $100 \%$ are well matched with the experiments [1] in low, medium and high frequency regions at appropriate $\mathrm{THz}$ fields. The free-carrier absorption is found to be mainly dictated by carrier-phonon, carrier lattice and carrier-carrier interaction mechanisms. Fig.4-a, -b, -c clearly demonstrate the microscopic valley transition behavior of electrons with interaction of different $\mathrm{THz}$ frequency pulses. In low frequency range and low peak $\mathrm{THz}$ field intensity, the acoustic scattering mechanism is found to dictate the intervalley carriers transfer. With the increase in peak $\mathrm{THz}$ field strength, the impact ionization scattering mechanism becomes dominant scattering mechanism which is shown from Fig. 5. The strong saturation absorption curve observed in Fig. 1 at high $\mathrm{THz}$ fields i.e. at $\sim 5$ and $15 \mathrm{MV} / \mathrm{m}$ can be successfully demonstrated through electrons impact ionization mechanisms. The increased number of electrons tends to enhance the absorption of $\mathrm{THz}$ photons in higher frequency range at higher peak field strength. On the other hand the increased number of electrons also increases the probability of the carrier scattering mechanisms. Hence, carrier generation due to impact ionization process and scattering processes compete with each other. Once the equilibrium attain between these two processes, the saturated behavior of absorption curve is observed under application of higher peak field strength. On other hand at low field strength, free carrier absorption is limited to free carriers only and we observe steep fall in absorption curve at higher frequency region i.e. (1.0- $2.0 \mathrm{THz})$.

Fig. 2 shows the complex conductivities at peak field intensities 1 (blue line), 5(red line) and $15 \mathrm{MV} / \mathrm{m}$ (black line) respectively with ramping $\mathrm{THz}$ frequencies. It is clearly reflected from the conductivity curves that at low peak $\mathrm{THz}$ field strength $(\sim 1 \mathrm{MV} / \mathrm{m})$, the conductivity steeply decreases with increasing the frequency from $0.2-2.0 \mathrm{THz}$. At moderate peak $\mathrm{THz}$ field intensity $(\sim 5 \mathrm{MV} / \mathrm{m})$, the conductivity is steeply deceases first in frequency range 0.2$1.0 \mathrm{THz}$ and in frequency range $1.0-2.0 \mathrm{THz}$, the conductivity curve attained the saturation. At higher peak $\mathrm{THz}$ field strength $(\sim 15 \mathrm{MV} / \mathrm{m})$, the conductivity curve shows strong saturation over entire frequency range i.e. $0.2-2.0 \mathrm{THz}$ range. The entire mechanisms at different $\mathrm{THz}$ field strengths are explained with the help of different scattering mechanisms with saturation phenomenon. At low $\mathrm{THz}$ field intensity, the acoustic (electron-phonon), intervalley and Coulomb scattering mechanisms are responsible for degrading the hot carriers drift velocity, whereas the free carrier absorption is limited to only available intrinsic carrier's density. Hence passing the low field strength single cycle $\mathrm{THz}$ pulse generates hot carriers, so initially the higher value of conductivity recorded. After some time free carrier absorption rate saturated due to limited number of free carriers available. However, continued scattering mechanisms cause the steep degradation in the conductivities values. It is well known that at $\geq 2 \mathrm{MV} / \mathrm{m}$, the impact ionization process initiated in $\mathrm{Ge}$ semiconductor and Coulomb (carrier-carrier) scattering mechanism is found to be dominant mechanism in moderate to 
high peak $\mathrm{THz}$ field strength. The high energy photons absorbed by the free electrons. With the increase in peak $\mathrm{THz}$ field strength above from threshold value, the impact ionization process started. The carriers generated due to impact ionization process take part in free carrier absorption mechanism. On the other hand due to increase number of free carriers generated, Coulomb scattering dominating over other scattering processes. The conductivity curve at peak $\mathrm{THz}$ field strength $5 \mathrm{MV} / \mathrm{m}$ and $15 \mathrm{MV} / \mathrm{m}$ justify the same. The saturated conductivity is observed for $15 \mathrm{MV} / \mathrm{m}$ case for entire frequency range (0.25-2.0 THz), Fig. 2 .

The free carrier absorption prompts to carrier's intervalley transitions. At frequencies $0.25 \mathrm{THz}$ at peak field strength $15 \mathrm{MV} / \mathrm{m}$ the number of electrons in each valley against applied single cycle $\mathrm{THz}$ pulse is shown in Fig. 3. It demonstrates the application of variation in $\mathrm{THz}$ field strength is responsible for variation in valley occupation ratio. These mechanisms are also reflected from the Fig. $4 a-4 b$ that the intervalley transitions are result of free carrier absorption. The L-valley electrons (the lowest energy state in Ge thin film) along with $\Gamma$-valley electrons absorb $\mathrm{THz}$ energy and excited to X-valley for positive and negative both half cycles of $\mathrm{THz}$ pulse (shown by black curve in Fig. 3). Once the THz pulse off, most of the X-valley electrons transfer itself to L-valley (lowest energy state) to attain the equilibrium.

The hot carriers transfer from lower energy state to higher energy state with respect to time is shown in Fig.4-a, b, c. The curves reflecting the carriers transition mechanisms at $0.25,0.75,1.25$ and $1.75 \mathrm{THz}$ frequencies at $\sim 15,5,1 \mathrm{MV} / \mathrm{m}$ peak $\mathrm{THz}$ field strengths respectively. It is evident from the curve that at lower frequency i.e. high pulse width, the carriers get enough time to interact strongly with the $\mathrm{THz}$ photons. Hence high intervalley carrier transitions are observed. However, with the increase in $\mathrm{THz}$ frequencies, the pulse width decreases, the interaction time of $\mathrm{THz}$ photon with free carriers also decreases, hence low intervalley transitions are obtained. It is observed that the lower to higher valley intervalley transitions are less prominent with the increase of the $\mathrm{THz}$ pulse frequency under peak field intensity $\sim 1 \mathrm{MV} / \mathrm{m}$ except at higher frequency i.e. $1.75 \mathrm{THz}$ as shown in Fig. 4c. The same results have been obtained for $\sim 15$ and $5 \mathrm{MV} / \mathrm{m}$ peak field intensities, Fig.4a and Fig 4 b. With increasing the $\mathrm{THz}$ frequency the $\mathrm{THz}$ pulse width which is in order of picosecond (ps), decreases, the intervalley transition rate decreases, the electrons get less time to interact with the $\mathrm{THz}$ field at higher frequencies range as compared to lower frequency ranges. The low $\mathrm{THz}$ frequencies are responsible for more intervalley transitions mechanisms justified the observation obtained here. At higher peak field strength, the high free carrier absorption rates are obtained as compared to lower peak field strengths. The free carrier generation due to impact ionization mechanism is responsible for observing high free carrier absorption. The degraded conductivity values obtained due to increased carrier-carrier (Coulomb) scattering rate at higher peak field strength.
The acoustic and coulomb scattering rates are compared in Fig.5. These curves are self explanatory. At the start of simulation i.e. in absence of $\mathrm{THz}$ pulses, the coulomb scattering initiated whereas acoustic scattering rates are almost negligible. The free carrier absorption causes to increase the carrier's energy hence the acoustic scattering rates are increased. The high energy $\mathrm{X}$-valley electrons show higher acoustic scattering rate as compared to $\Gamma$ and L-valley respectively. The effect of $\mathrm{THz}$ pulse is negligible over Coulomb scattering rates, which is almost saturated as the carrier energy increases. The impact ionization scattering rate is shown in Fig, 6 with respect to $\mathrm{THz}$ field strength. It is clearly reflected from the curve that the threshold field value for impact ionization process is $\sim 1.2 \mathrm{MV} / \mathrm{m}$. The free electrons created due to impact ionization process start contributing for the free carrier absorption process.

\section{Conclusion}

In current manuscript, the authors have successfully calibrated the real time $\mathrm{THz}$ spectroscopy experiments without using any fitting parameters. The proper understanding of hot carriers dynamics on the basis of solid physical foundation is carried out. The significant contributions of the various scattering mechanisms at atomistic scale on the complex conductivity and free carrier absorption have been observed and successfully demonstrated. The in-house developed algorithms is converted into innovative TNL-TS simulator which is capable to demonstrate the experimental $\mathrm{THz}$ spectroscopy characterization method with proper understanding of ultrafast carriers dynamics with various scatterings saturation phenomenon.

AVAILABILITY OF DATA: The data that support the findings of this study are available from the corresponding author upon reasonable request.

\section{References:}

1. János Hebling, Matthias C. Hoffmann, Harold Y. Hwang, Ka-Lo Yeh and Keith A. Nelson, Observation of non equilibrium carrier distribution in $\mathrm{Ge}, \mathrm{Si}$, and $\mathrm{GaAs}$ by terahertz pump-terahertz probe measurements, Phys Rev B 81, 035201 (2010).

2. James Lloyd-Hughes, PM Oppeneer, T Pereira Dos Santos, Andre Schleife, Sheng Meng, Michael A Sentef, Michael Ruggenthaler, Angel Rubio, Ilie Radu, Margaret Murnane, Xun Shi, Henry Kapteyn, Benjamin Stadtmüller, Keshav M Dani, FH da Jornada, Eva Prinz, Martin Aeschlimann, RL Milot, Maria Burdanova, Jessica Boland, T Cocker, F Hegmann, The 2021 ultrafast spectroscopic probes of condensed matter roadmap, J. Phys.: Condens. Matter 33, 353001(2021).

3. Jens Neu and Charles A. Schmuttenmaer, Tutorial: An introduction to terahertz time domain spectroscopy (THz-TDS), J. Appl. Phys. 124, 231101 (2018).

4. Benjamin Reinhard, Klemens M. Schmitt, Viktoria Wollrab, Jens Neu, René Beigang, and Marco Rahm, Metamaterial near-field sensor for deep-subwavelength thickness measurements and sensitive refractometry in the terahertz frequency range, Appl. Phys. Lett. 100, 221101 (2012).

5. Eugene A. Mashkovich, Alexander I. Shugurov, Shipei Ozawa, Elmer Estacio, Masahiko Tani, and Michael I. Bakunov, Noncollinear ElectroOptic Sampling of Terahertz Waves in a Thick GaAs Crystal, IEEE Trans. THz Science \& Tech. 5(5), 732 (2015). 
6. T. L. Cocker, D. Baillie, M. Buruma, L. V. Titova, R. D. Sydora, F. Marsiglio, and F. A. Hegmann, Microscopic origin of the Drude-Smith model, Phys. Rev. B 96, 205439 (2017).

7. S. Bonetti, M. Hoffmann, M.-J. Sher, Z. Chen, S.-H. Yang, M. Samant, S. S. P. Parkin, and H. A. Durr, THz-Driven Ultrafast Spin-Lattice Scattering in Amorphous Metallic Ferromagnets, Phys. Rev. Lett. 117, 087205 (2016).

8. J. Clerouin, P. Noiret, V. N. Korobenko, and A. D. Rakhel, Direct measurements and ab initio simulations for expanded fluid aluminum in the metal-nonmetal transition rangePhys. Rev. B 78, 224203 (2008).

9. Silaeva E, B'evillon E, Stoian R and Colombier J P, Ultrafast electron dynamics and orbital-dependent thermalization in photoexcited metals, Phys. Rev. B 98094306 (2018).

10. Sjakste J, Tanimura K, Barbarino G, Perfetti L and Vast N, Hot electron relaxation dynamics in semiconductors: assessing the strength of the electron-phonon coupling from the theoretical and experimental view points, J. Phys.: Condens. Matter 30 353001(2018).

11. Zhiyang $\mathrm{Wu}$, Weining Liu, Shuang Wang, Junfeng Jiang, Tianhua Xu, Xun Yu, Tiegen Liu, RETRACTED: Terahertz and infrared refractive index of a Ge-core optical fiber micro-spheresOptics Communications 474, 126104 (2020).

12. I. A. Kaplunov, A. I. Kolesnikov, G. I. Kropotov, and V. E. Rogalin, Optical Properties of Single-Crystal Germanium in the THz Range, Optics and Spectroscopy 126( 3), 191 (2019).

13. TNL-TS Simulator user manual (2021), Tech Next Lab Private Limited https://www.technextlab.com/sp.html

14. A. Srivastava, A. Saxena, P. K. Saxena, F. K.Gupta, P. Shakya, P. Srivastava, M. Dixit, S.Gambhir, R. K. Shukla \& A. Srivastava, An innovative technique for electronic transport model of group-III nitrides, Scientific Reports 10, 18706 (2020).

15. Takuya Maeda, Tetsuo Narita, Shinji Yamada, Tetsu Kachi, Tsunenobu Kimoto, Masahiro Horita, and Jun Suda, Impact Ionization Coefficients in $\mathrm{GaN}$ Measured by Above- and Sub-Eg Illuminations for $\mathrm{p}-/ \mathrm{n}+$ Junction, IEDM Tech. Digest, 4.2.1(2019)

16. Mowfaq Jalil Ahmed, Temperature dependence of electron impact ionization coefficient in bulk silicon AIP Conference Proceedings 1888, 020034 (2017).

17. Dong Ji and Srabanti Chowdhury, On impact ionization and avalanche in gallium nitride, Appl. Phys. Lett. 117, 252107 (2020).

18. Adachi, S. In: (eds Kasap, S. \& Capper, P.) Springer Handbook of Electronic and Photonic Materials, Springer Handbooks. (Springer, Cham, 2017).

19. Xiaoyu, W. \& Erez, B. Scattering mechanisms and electrical transport near an Ising ematic quantum critical point, Phys. Rev. B 99, 235136 (2019).

20. Jacoboni, C. \& Lugli, P. The Monte Carlo Method for Semiconductor Device Simulation (Springer, New York, 2019)

21. D. Chattopadhyay and A. Ghosal, Electron scattering in heavily doped compensated polar semiconductors, Phys. Rev. B 25(10), 6538 (1982).

22. J. Zhang, Piezoelectric effect on the thermal conductivity of monolayer gallium nitride, J. Appl. Phys. 123, 035102 (2018).

23. Alex M. Ganose, Junsoo Park, Alireza Faghaninia, Rachel WoodsRobinson1, Kristin A. Persson \& Anubhav Jain, Efficient calculation of carrier scattering rates from first principles, Nature Communications $12,2222(2021)$.

\section{$\underline{\text { List of Figures }}$}

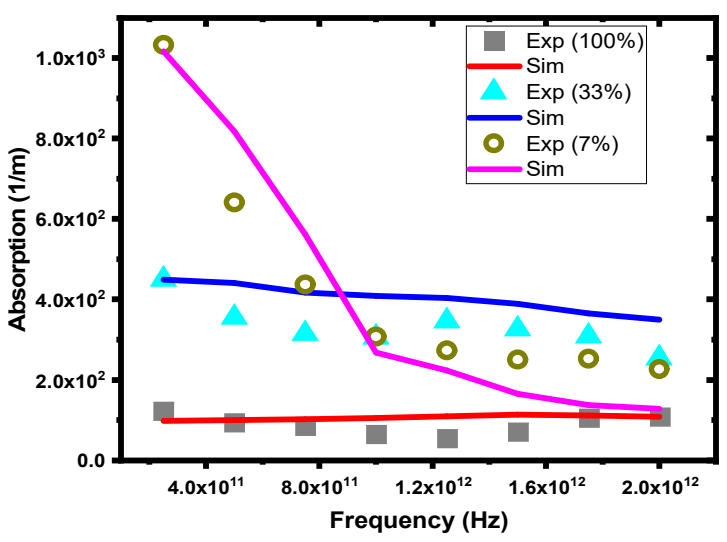

Fig.1. Absorption spectrum at peak THz intensity 1 (7\%), 5 (33\%), 15 (100\%) MV/m with varying THz Frequency. Data from present work is displayed as solid lines where as experimental results taken from reference [1] are depicted by circles, triangles and squares respectively.

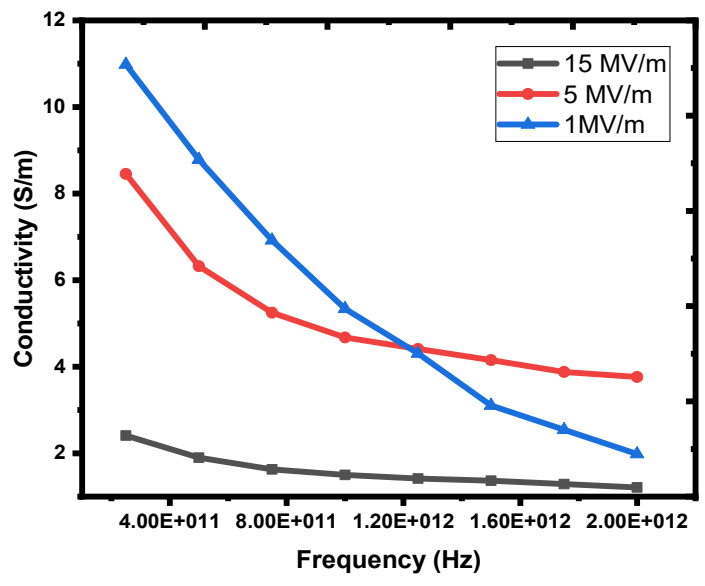

Fig.2. Complex conductivities at Peak THz Intensity 1, 5, $15 \mathrm{MV} / \mathrm{m}$ with ramping THz Frequency

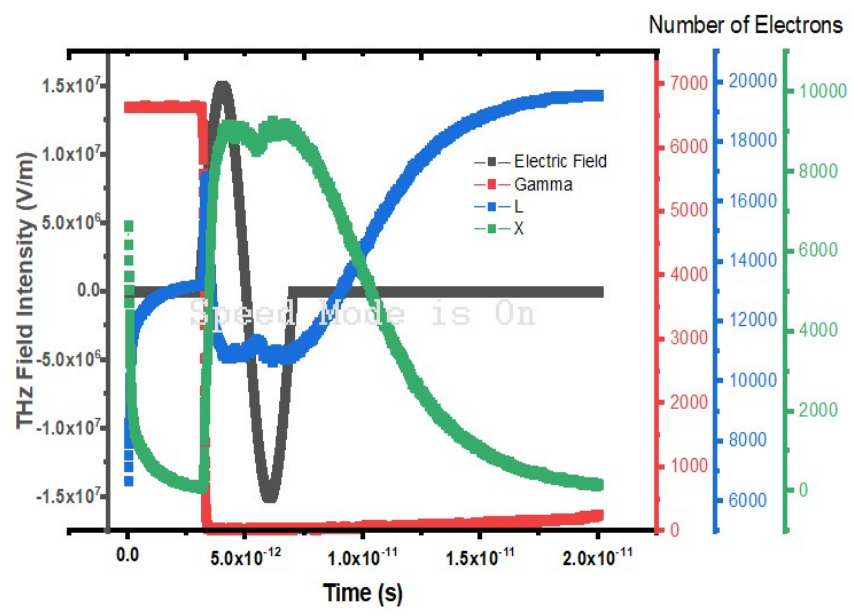


Fig.3. Variation in $\mathrm{THz}$ field Intensity with transition in number of electrons of $\Gamma, \mathrm{L}$ and $\mathrm{X}$ valley due to interaction of $\mathrm{THz}$ field at $0.25 \mathrm{THz}$ frequency with respect Time
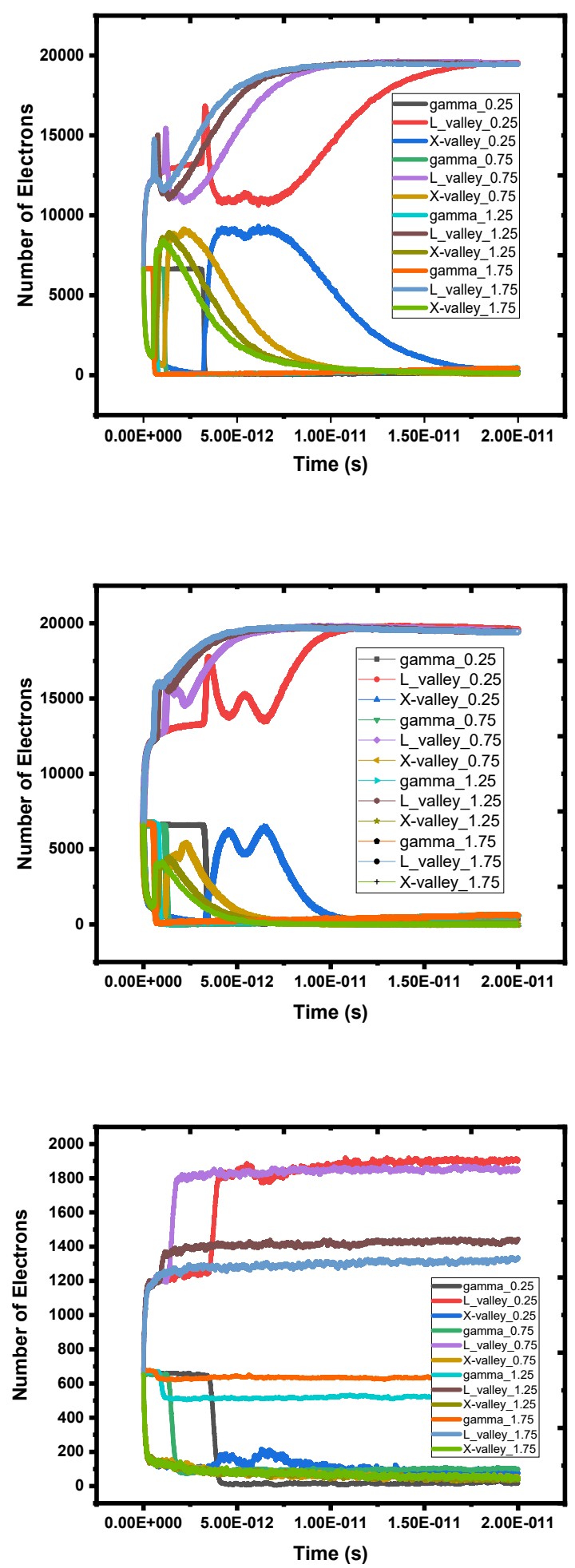

Fig.4-a, 4-b, 4-c. Variation in Valley Population at various $\mathrm{THz}$ Frequencies at peak field intensity 15, 5 and $1 \mathrm{MV} / \mathrm{m}$ with respect Time respectively

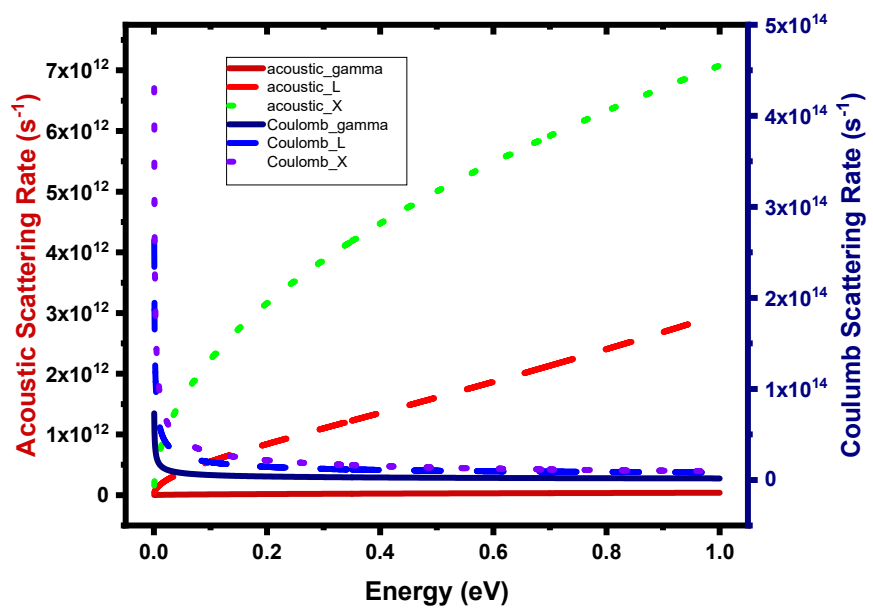

Fig.5. Acoustic (electron-phonon) and Coulomb (carriercarrier) scattering rate with respect to Energy $(\mathrm{eV})$.

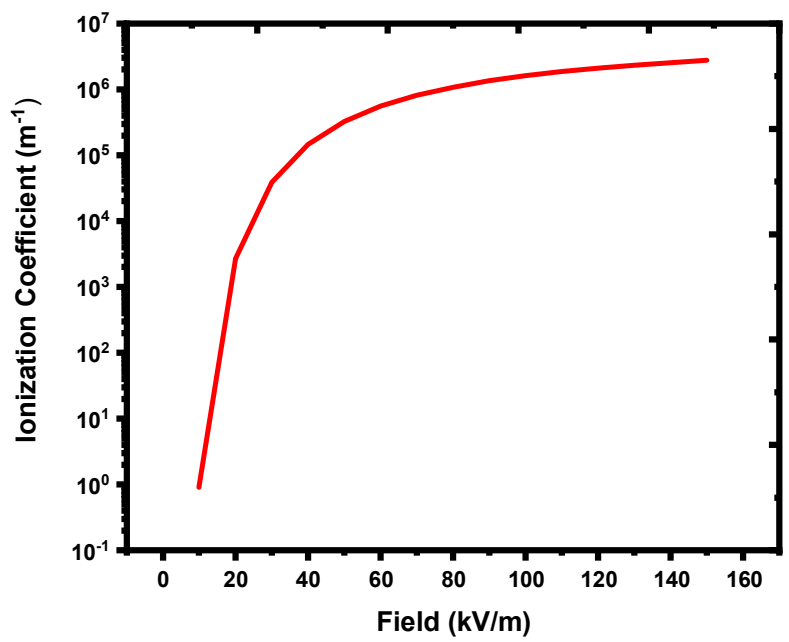

Fig.6. Variation of impact ionization coefficient with respect to varying THZ field intensity 
Table I Ge Material Parameters used in ultrafast hot carrier dynamics simulation

$\mathrm{M}$ - mass density $v_{s}$ - Sound velocity, $D_{a}$-acoustic deformation potential, $\varepsilon_{\mathrm{s}}$-static dielectric constant, $\varepsilon_{\infty}-$ High Frequency dielectric constant, $D_{i j}$ - Intervalley Deformation Potentials, $\alpha$ - non-parabolicity factor, are taken from reference [18].

$\mathrm{m}_{\mathrm{r}}$ - Effective mass at $\Gamma$ valley, $\mathrm{m}_{\mathrm{L}}-$ Effective mass at $\mathrm{L}$ valley, $\mathrm{m}_{\mathrm{X}^{-}}$Effective mass at $\mathrm{X}$ valley, $L-\Gamma, X-\Gamma$ are valley separation, $\hbar \omega_{L O}$-Optical Phonon energies, $\hbar \omega_{i j}-$ Intervalley Phonon Energies are extracted from the E-k data obtained through FullBand simulator.

\begin{tabular}{|c|c|c|}
\hline \multicolumn{2}{|c|}{ Parameters } & Values Taken \\
\hline \multicolumn{2}{|c|}{$\mathrm{M}\left(\mathrm{kg} / \mathrm{m}^{3}\right)$} & 5320 \\
\hline \multicolumn{2}{|c|}{$\mathrm{v}_{\mathrm{s}}(\mathrm{m} / \mathrm{s})$} & 5400 \\
\hline \multicolumn{2}{|c|}{$\mathrm{D}_{\mathrm{a}}(\mathrm{eV})$} & 2.5 \\
\hline \multicolumn{2}{|l|}{$\varepsilon_{s}$} & 16.2 \\
\hline \multicolumn{2}{|l|}{$\varepsilon_{\infty}$} & 16.2 \\
\hline \multicolumn{2}{|l|}{$m_{\Gamma}$} & 0.036 \\
\hline \multicolumn{2}{|l|}{$m_{\mathrm{L}}$} & 0.455 \\
\hline \multicolumn{2}{|l|}{$\mathrm{m}_{\mathrm{X}}$} & 1.21 \\
\hline \multicolumn{2}{|c|}{$L-\Gamma(\mathrm{eV})$} & -0.14 \\
\hline \multicolumn{2}{|c|}{$X-\Gamma(\mathrm{eV})$} & 0.635 \\
\hline \multirow{3}{*}{$\begin{array}{l}\text { Equivalent } \\
\text { valleys }\end{array}$} & $\bar{\Gamma}$ & 1 \\
\hline & $\mathrm{L}$ & 4 \\
\hline & $\mathrm{X}$ & 3 \\
\hline \multicolumn{2}{|c|}{$\hbar \omega_{L O}(\mathrm{meV})$} & 0.23 \\
\hline \multicolumn{2}{|c|}{$D_{i j}\left(\mathrm{eV} \mathrm{m}^{-1}\right)$} & $2 \mathrm{E} 10$ \\
\hline \multicolumn{2}{|c|}{$\hbar \omega_{i j}(\mathrm{meV})$} & 37 \\
\hline \multirow[t]{3}{*}{$\alpha\left(\mathrm{eV}^{-1}\right)$} & $\Gamma$ & 0 \\
\hline & $\mathrm{L}$ & 0.3 \\
\hline & $\mathrm{X}$ & 0 \\
\hline \multicolumn{2}{|c|}{ Doping $\left(\mathrm{m}^{-3}\right)$} & $2 \mathrm{E} 21$ \\
\hline
\end{tabular}

Appendix A

An accurate comparison between experimental carrier mobility and theoretical calculation is of great importance for the determination of a variety of fundamental material parameters and carrier scattering mechanisms. The various carrier scattering mechanisms used in current work is given as [20]-[23]

1. Acoustic Phonon scattering [20] -[23]

$$
\tau(E)=\frac{2 \pi D_{a c}^{2} k_{B} T}{\hbar C_{l}}\left(\frac{\left(2 m_{d}\right)^{3 / 2} \sqrt{E(1+\alpha E)}}{4 \pi^{2} \hbar^{3}}\right)(1+2 \alpha E)
$$

2. Intervalley Phonon Scattering [20] -[23]

$$
\begin{aligned}
\tau(E)=\left(\frac{\pi D_{i j}^{2} Z_{j}}{\rho W_{i j}}\right) & *\left(n\left(W_{i j}\right)+\frac{1}{2} \mp \frac{1}{2}\right) \\
& *\left(\frac{\left(2 m_{d}\right)^{3 / 2} \sqrt{E_{f}\left(1+\alpha E_{f}\right)}}{4 \pi^{2} \hbar^{3}}\right)\left(1+2 \alpha E_{f}\right)
\end{aligned}
$$

Here, $E_{f}=E \pm \hbar W_{i j}-\Delta E_{i j}$

3. Ionised Impurity Scattering [22]- [23]

$$
\begin{array}{r}
\tau(E)=\left[\frac{\sqrt{2} e^{4} N_{l} m_{d}^{3 / 2}}{\pi \varepsilon_{s}^{2} \hbar^{4}}\right] *(\sqrt{E(1+\alpha E}) *(1+2 \alpha E) \\
*\left[\frac{1}{q_{D}^{2}\left(q_{D}^{2}+\frac{8 m_{d} E(1+2 \alpha E}{\hbar^{2}}\right)}\right]
\end{array}
$$

Here $q_{D}=\sqrt{\frac{e^{3} N_{I}}{\varepsilon k_{B} T}}$

4. Impact Ionization Scattering [15] - [17]

$$
1 / \tau_{i i}(E)= \begin{cases}\frac{\left(E-E_{t h}(T)\right)^{2}}{\tau_{p}(E) E_{t h}^{2}(T)} ; \quad E>E_{t h}(T) \\ \frac{\left(E-E_{g}(T)\right)^{3.5}}{\tau_{p}(E) E_{g}^{3.5}(T)} ; & E_{g}<E<E_{t h}\end{cases}
$$

Here $1 / \tau_{\mathrm{p}}$ is total phonon scattering rate, $\mathrm{E}$ is the carrier energy, $E_{t h}$ is the temperature dependent threshold energy of impact ionization and is given as

$E_{t h}(T)=\frac{E_{g}(T)}{E_{g}(300 K)} E_{t h}(300 K)$

The ionization coefficient $\alpha_{n}(E)$, is given by Chynoweth's empirical formula;

$\alpha_{n}(E)=2.69 \times 10^{7} \exp \left(-\frac{2.27 \times 10^{7}}{\epsilon}\right)$ 